\title{
Estimation of Hysteretic Interfacial Stiffness of Contact Surfaces
}

\author{
Nohyu Kim
}

\begin{abstract}
This paper proposes an ultrasonic method for measurement of linear and hysteretic interfacial stiffness of contacting surfaces between two steel plates subjected to nominal compression pressure. Interfacial stiffness was evaluated by the reflection and transmission coefficients obtained from three consecutive reflection waves from solid-solid surface using the shear wave. A nonlinear hysteretic spring model was proposed and used to define the quantitative interfacial stiffness of interface with the reflection and transmission coefficients. Acoustic model for 1-D wave propagation across interfaces is developed to formulate the reflection and transmission waves and to determine the linear and nonlinear hysteretic interfacial stiffness. Two identical plates are put together to form a contacting surface and pressed by bolt-fastening to measure interfacial stiffness at different states of contact pressure. It is found from experiment that the linear and hysteretic interfacial stiffness are successfully determined by the reflection and transmission coefficient at the contact surfaces through ultrasonic pulse-echo measurement.
\end{abstract}

Keywords: Hysteretic Interfacial Stiffness, Contact Surface, Contact Nonlinearity, Ultrasound

\section{Introduction}

The physical nature of the contact acoustic nonlinearity (CAN) has been explained and analyzed by micro-mechanical models for contacttype interface. The variation of contact area due to the deformation of asperities is known to cause the nonlinear elasticity of interfaces. In the previous works, this interface is considered as a nonlinear spring whose stiffness is proportional to the contact area [1-5].

Non-invasive evaluation of the contact condition is important not only for nondestructive test but for the tribology and the design of mechanical components with contacting interfaces. Micromechanical behavior of the contacting surfaces is so complicated about nonlinearity that it is hardly understood by micro-scale properties of the solids. Instead, it can be effectively explained in macro-scale by the interfacial stiffness which is employed to model the nonlinear stressdisplacement relation of the contact surfaces in nonlinear spring models. This interfacial stiffness is known to offer a lot of useful information on the nature of contact interface, for example, the detection tool of a closed crack that does not produce linear scattering waves. Ultrasound is an attractive tool for monitoring this contact condition between solid components because of its penetration power and sensitivity to the discontinuity [6].

Interfacial stiffness of contacting surfaces has been determined by different principles such as the normal and oblique reflection of bulk waves or the velocity/attenuation of guided waves. Biwa [1-3] evaluated the normal stiffness and the tangential stiffness of contacting poly(methylmethacrylate) (PMMA) blocks from both bulk wave reflection and interface wave velocity measurements. However, all the investigations conducted by previous researches are to measure the linear interfacial stiffness only, which is a part of the characteristics of the interface. Even though the linear stiffness is the most important property of contact interface in dynamic behavior of solid-solid contact surface, the nonlinear 
hysteresis of contact surfaces plays a great role in the irreversible acoustic property of the interface. This has been an issue in contact nonlinear acoustics, but not fully understood yet.

In this paper, a new measurement technique to estimate the nonlinear hysteretic interfacial stiffness at the interface is presented using only one transducer without through-transmission test. Multiple transmissions and reflections across the interface are generated to extract the information on the reflection and transmission coefficients which is associated with interfacial stiffness by theoretical model developed for contacting interface. Reflection and transmission coefficients from consecutive reflected waves from the interface of two contacting plates are calculated and substituted to the acoustic model to calculate the hysteretic interfacial stiffness. For the demonstration of the stiffness measurement, a solid-solid interface is constructed using steel plates and inspected by $5 \mathrm{MHz}$ shear transducer to measure the reflection and transmission waves across the interface at various pressures. The experimental results are represented and discussed to verify the method proposed in this paper.

\section{Interfacial Stiffness in Solid-Solid interface}

At the micro-scale, the contact interface appears as two surfaces of irregular topology which intersect to form micro-void spaces and asperities of contact. The interface loaded in shear or compression exhibits a highly nonlinear stress-displacement relationship resulting from plastic deformation of the asperities. The hysteresis is also accompanied with the classical nonlinear elasticity as shown in the stressdisplacement curve in Fig. 1 indicating the presence of inelastic deformation of the asperities of contact and frictional sliding between contacting boundaries[7]. Those features of contact surface play an important role in the interaction with elastic waves. The interface stiffness is the quantity that relates the displacement of the interface to the traction of closed contact interfaces, which may be linear or nonlinear.

Let us consider a nonlinear hystereticoscillatory cycle $S_{L}-S_{U}$ between two deformation states, $u_{1}$ and $u_{2}$, in Fig. 1 caused by acoustic waves. The irreversible deformation starts from $u_{1}$ to $u_{2}$ following a loading path $S_{L}$ and returns to the original point $u_{1}$ via an unloading path $S_{L}$ completing one cycle of motion. A linear deformation path $\mathrm{S}$ from $u_{1}$ to $u_{2}$ is also presented in Fig. 1 to introduce the linear stiffness $K_{z}$ from the proportional linearity. During the loading process $S_{L}$ of this hysteresis cycle, the work done to the medium by stress is larger than that of the linear loading $\mathrm{S}$, while the work done by the medium during the unloading process $S_{U}$ is smaller than that of the linear unloading. The amount of the energy difference is dissipated by the hysteresis of contact interfaces. In these processes, the contact interface works as a non-classical hysteretic spring that gets stiffer during loading and softer for unloading. Thus this characteristic of the hysteretic spring can be formulated by adding a supplementary stiffness to the linear spring during loading, and subtracting the same stiffness from the linear spring during unloading[7]. Such a hysteretic spring force can be considered as Coulomb friction force and/or viscous force. In this paper, a hysteretic nonlinear stiffness $K_{n z}$ is defined such that the hysteretic force is linearly proportional to the displacement $\Delta u=\left(u_{2}-u_{1}\right)$. The hysteretic nonlinear stiffness $K_{n z}$ is complex and can be determined by the energy loss dissipated by one cycle of the process $S_{L}-S_{U}$. Based on these assumptions, the stress-displacement relation across the interface in the process $S_{L^{-}} S_{U}$ is expressed by the superposition of a linear spring $K_{z}$ and nonlinear hysteretic spring $K_{n z}$ as 
follows,
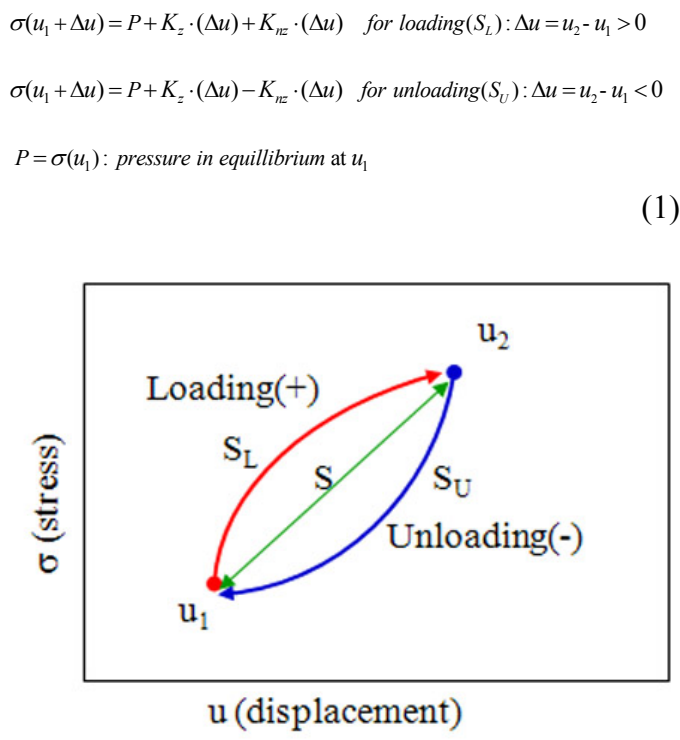

Fig. 1 Hysteretic behavior of contact interfaces during loading-unloading process

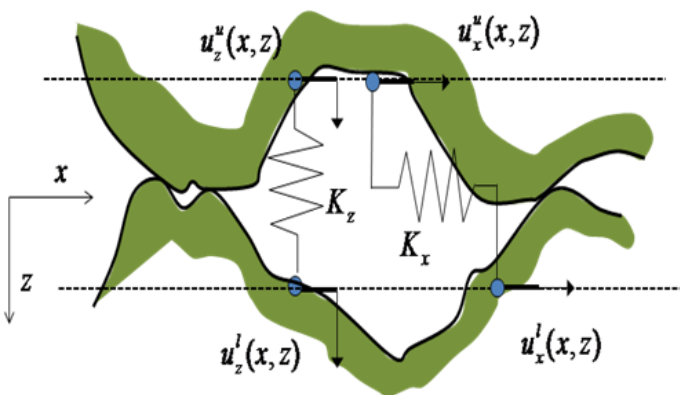

Fig. 2 A spring model for contact surfaces

Now suppose that two elastic bodies with identical material properties are put into contact by static pressure $P$. Due to the surface roughness, the contact at the interface forms a microscopically imperfect elastic-plastic deformation between the upper (denoted by superscript $u$ ) and lower (denoted by superscript $l$ ) rough surfaces as shown in Fig. 2. The displacement vectors of the upper and lower surface are given in Fig. 2 by $u^{u}=\left(u_{x}^{u}, u_{z}^{u}\right)$ and $u^{l}=\left(u_{x}^{l}, u_{z}^{l}\right)$. Since both the displacement and the stress have discontinuity across the interface, some boundary conditions are necessary to connect those discontinuities. These conditions are obtained from the constitutive properties of the interface formulated by the hysteretic nonlinear spring $K_{n z}$ in Eq. (1).

Consider a longitudinal plane wave of wave number $K$ and frequency $\omega$ incident to the contact interfaces in $z$-direction in Fig. 2.

Then the incident harmonic wave $\left(u_{z}^{u}(z, t)\right)_{i}=A e^{i \kappa(z-c t)}$ from the upper medium generates a reflected wave $\left(u_{z}^{u}(z, t)\right)_{r}=B e^{-i \kappa(z+c t)}$ and a transmitted wave $\left(u_{z}^{l}(z, t)\right)_{t}=C e^{i \kappa(z-c t)}$ at the interface. The amplitudes of the waves denoted as A, B, and $\mathrm{C}$ are a complex magnitude, and the superscripts, $u$ and $l$, represent the upper and lower medium of the interface, and the subscripts, $r$ and $t$, mean the reflection and transmission, respectively. Then, the complex transmission and reflection coefficient $\mathrm{T}$ and $\mathrm{R}$ for displacement are given at the interface $\mathrm{z}=0$ by [7],

$$
\begin{gathered}
R(\text { Reflection Coeff } .)=\frac{\left(u_{z}^{u}(0, t)\right)_{r}}{\left(u_{z}^{u}(0, t)\right)_{i}}=\frac{2 i\left(\frac{K_{n z}}{Z \omega}\right)+1}{2 i\left(\frac{K_{z}}{Z \omega}\right)-1} \\
T(\text { Transmission Coeff } .)=\frac{\left(u_{z}^{l}(0, t)\right)_{t}}{\left(u_{z}^{u}(0, t)\right)_{i}}=\frac{2 i \frac{\left(K_{z}-K_{n z}\right)}{Z \omega}}{2 i\left(\frac{K_{z}}{Z \omega}\right)-1}
\end{gathered}
$$

where, $Z=\rho c$ is the acoustic impedance, $\omega$ is the angular velocity, and $K_{z}, K_{n z}$ are the linear and hysteretic interfacial stiffness in z-direction defined in Eq. (1). Therefore, $K_{z}$ and $K_{n z}$ are determined by solving the simultaneous equations in Eq. (2), which are simply given in terms of the reflection and transmission coefficients, $\mathrm{R}$ and $\mathrm{T}$, at contact interface as followings,

$$
\begin{aligned}
& R^{2}=\frac{4\left(\frac{K_{n z}}{Z \omega}\right)^{2}+1}{4\left(\frac{K_{z}}{Z \omega}\right)^{2}+1} \text { or } \frac{K_{z}}{Z \omega} \approx \frac{\sqrt{1-\mathrm{R}^{2}}}{2 \mathrm{R}} \text { for a small } \frac{K_{n z}}{K_{z}} \\
& T^{2}=\frac{4\left(\frac{K_{z}}{Z \omega}-\frac{K_{n z}}{Z \omega}\right)^{2}}{4\left(\frac{K_{z}}{Z \omega}\right)^{2}+1} \text { or } \frac{K_{n z}}{Z \omega} \approx \frac{2 \frac{4\left(\frac{K_{z}}{Z \omega}\right)^{2}}{4\left(\frac{K_{z}}{Z \omega}\right)^{2}+1}-T^{2}}{2 \frac{4\left(\frac{K_{z}}{Z \omega}\right)}{4\left(\frac{K_{z}}{Z \omega}\right)^{2}+1}} \text { for a small } \frac{K_{n z}}{K_{z}}
\end{aligned}
$$


In Eq. (3), the terms, $\frac{K_{z}}{Z w}$ and $\frac{K_{n z}}{Z w}$, are the specific linear and hysteretic interfacial stiffness, a dimensionless quantities.

\section{Measurement of Reflection and Transmission Coefficients}

When a normal incident wave $u_{i}$ is applied to solid-solid contact interface of two identical plates by a transducer as shown in Fig. 3(a), three reflection waves from the plates are generated consecutively as $\mathrm{L}_{1}, \mathrm{~L}_{2}$, and $\mathrm{L}_{3}$ in order. $\mathrm{L}_{1}$ is the first arriving wave reflected once directly from the interface between the plates, while $\mathrm{L}_{2}$ and $\mathrm{L}_{3}$ are reflected waves traveling two times and three-times of the distance the wave $\mathrm{L}_{1}$ does. Therefore, the round-trip distance of $\mathrm{L}_{1}$ is two times of the thickness of the plate, $2 \mathrm{~d}$, and $\mathrm{L}_{2}$ for $4 \mathrm{~d}, \mathrm{~L}_{3}$ for

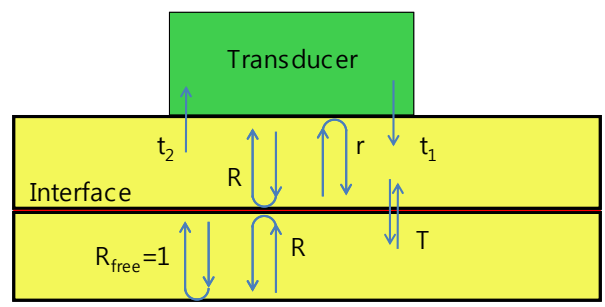

Free surface

(a)

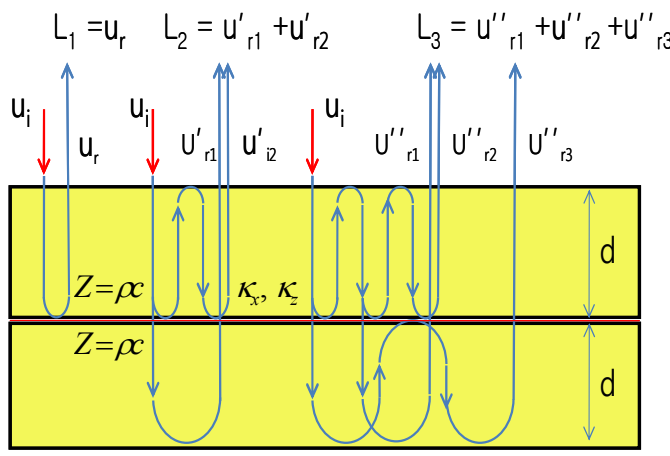

(b)

Fig. 3 Multiple reflection waves from interface, (a) the transmission and reflection coefficients of interfaces, (b) compositions of $L_{1}, L_{2}, L_{3}$ 6d. While $L_{1}$ is composed of only one reflection wave $u_{r}$ as shown in Fig $3(b), L_{2}$ and $L_{3}$ have two and three reflection wave components. $\mathrm{L}_{2}$ is the sum of two kinds of waves, which are $u_{r 1}^{\prime}+u_{r 2}^{\prime}$. $\mathrm{L}_{3}$ is $u_{r 1}^{\prime \prime}+u_{r 2}^{\prime \prime}+u_{r 3}^{\prime \prime}$. In Fig. 3(a), all transmission and reflection coefficients between the transducer and plate are priori-known and noted by $\mathrm{r}, t_{1}$, and $t_{2}$ as represented in Fig. 3(a), except the unknown $\mathrm{R}$ and $\mathrm{T}$ at the interface of interest.

Then the reflection and transmission coefficient, $\mathrm{R}$ and $\mathrm{T}$ of the interface can be expressed by the magnitude of the waves $\mathrm{L}_{1}, \mathrm{~L}_{2}$, and $\mathrm{L}_{3}$ [7].

$$
\frac{\left|L_{3}\right|}{\left|L_{1}\right|} /\left(\frac{\left|L_{2}\right|}{\left|L_{1}\right|}\right)^{2}=\frac{R^{2}\left|R^{2}(r+1)^{2}-(2 r+1)\right|}{\left|R^{2}(r+1)-1\right|^{2}}
$$

Eq. (4) shows that the reflection and transmission coefficients, $\mathrm{R}$ and $\mathrm{T}$, at contact interfaces can be simply obtained and substituted to Eq. (3) for the calculation of the linear and nonlinear hysteretic interfacial stiffness.

\section{Experiment and Results}

In order to demonstrate the method proposed in this paper, a solid-solid interface is created artificially by clamping two steel plates by high-tension bolts as shown in Fig. 4(a). Before joining two plates, they are ground flat well and smooth enough to get an area-contact instead of line-contact. The plate specimen has a hexagonal washer-shape of $50 \mathrm{~mm}$ in outer diameter and $25 \mathrm{~mm}$ in inner diameter with $10 \mathrm{~mm}$ thickness. A mounting jig to hold ultrasonic transducer on the specimen is also made and set on the top surface of the steel plates as shown in Fig. 4(b).

Two identical hexagonal washer-type plates were initially put together by hands, so that only a small amount of pressure is imposed on the interface of the plates. At this negligible pressure, shear ultrasonic wave was sent to the 
interface from the top of the specimen. Then reflected waves $L_{1}, L_{2}$, and $L_{3}$ from the interface are selected and saved for analysis and measurement of interfacial stiffness. In experiment, for convenience of calculation, $\mathrm{L}_{2}$ and $\mathrm{L}_{3}$ are all normalized by $\mathrm{L}_{1}$ and inserted into Eq. (4) to obtain reflection coefficient $R$ first. The value of $r$ on the right side of Eq. (4) was set to 0.16 , which is the theoretical reflection coefficient between PZT and steel.

The specimen was tightened by torque wrench in order to apply compression load to the interface. The torque is increased from zero up to $75 \mathrm{Nm}$ by $10 \mathrm{Nm}$. At each load, the contact interface of the specimen was examined at the same position by pulse-echo tests to measure the reflection and transmission energy from the interface using pulse shear wave (5 MHz) as described in Fig. 4(b). The amplitudes of first three consecutive reflection signals, $\mathrm{L}_{1}, \mathrm{~L}_{2}, \mathrm{~L}_{3}$, were detected and normalized to calculate $\frac{\left|L_{2}\right|}{\left|L_{1}\right|}$ and $\frac{\left|L_{3}\right|}{\left|L_{1}\right|}$, by which the reflection and transmission coefficient, $\mathrm{R}$ and $\mathrm{T}$, were determined using Eq. (3). These $\mathrm{R}$ and $\mathrm{T}$ values are again put into Eq. (4) to get interfacial stiffness at different load conditions. Experimental results for the measurement of interfacial stiffness at different levels of pressure are summarized in Table 1, where reflection/

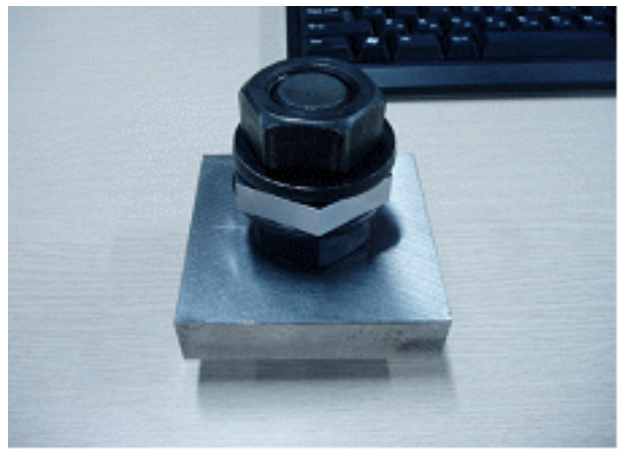

(a) transmission coefficients are listed with interfacial stiffness. It is observed that the interfacial stiffness increases with the compression load indicating a nonlinearity and hysteresis. These nonlinear characteristics are represented again by graph in Fig. 5, where a big jump in the stiffness can be observed at the beginning of contact from zero to $5 \mathrm{Nm}$ of clamping torque. After the region, both of the stiffness increase with the increase of clamping force with a different increasing rate. The linear stiffness increases more rapidly than the hysteretic stiffness. However, the ratio of the hysteretic stiffness to the linear interfacial stiffness decreases as the contact pressure increases, which means that the linear interfacial stiffness becomes more important than the hysteretic stiffness for the interface that is tightly pressed. It is also important that the hysteretic interfacial stiffness can be evaluated by a simple ultrasonic measurement of reflection and transmission coefficients at the contact surface, because the interfacial stiffness including the linear and hysteretic stiffness tells about the characteristic or the state of contact surface, for example, whether the boundary surfaces are contacting tightly or open. If the linear interfacial stiffness $K_{z}$ goes to zero, the reflection coefficient of the interface approaches unity, leading to a relatively large hysteresis on the contact surface.

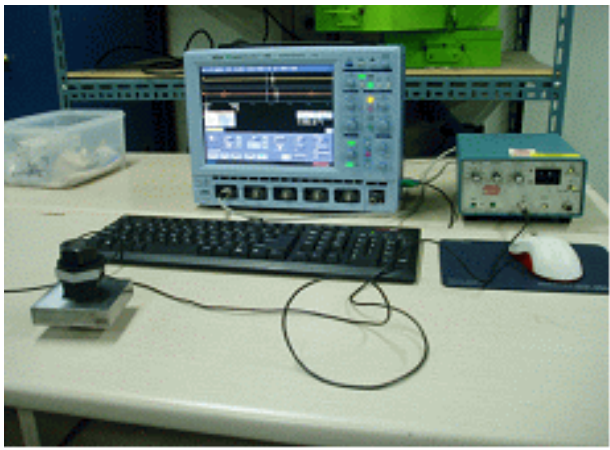

(b)

Fig. 4 Experimental set-up for the measurement of multiple reflection waves from the interface. (a) hexagonal plates pressed together to form a contact surface, (b) pulse-echo test 


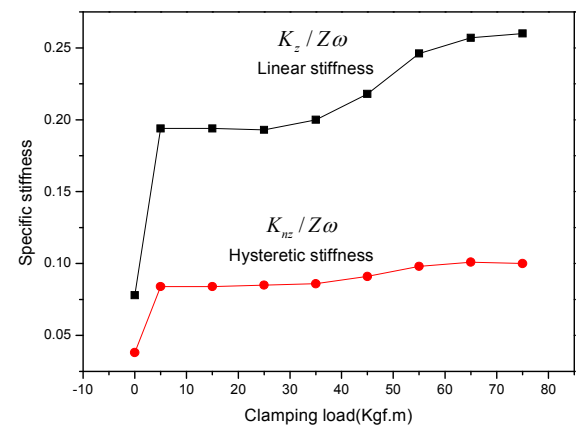

Fig. 5 Variation of the linear and hysteretic interfacial stiffness with the clamping force of the specimen

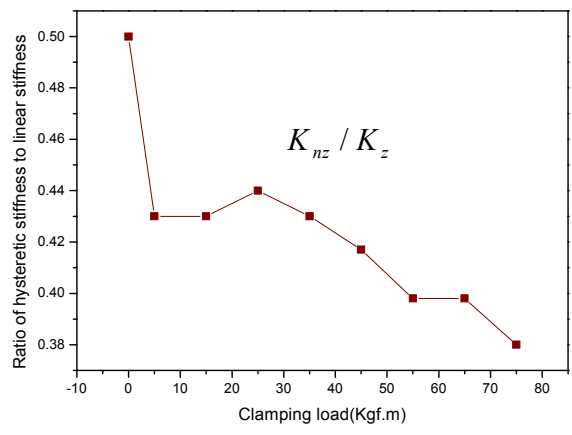

Fig. 6 Variation of the stiffness ratio according to the clamping force

Table 1 Measurement results

\begin{tabular}{|c|c|c|c|c|c|c|}
\hline $\begin{array}{c}\text { Clamping } \\
\text { Torque } \\
(\mathrm{Nm})\end{array}$ & $\begin{array}{l}\text { Reflection } \\
\text { Coefficient } \\
\text { (R) }\end{array}$ & $\begin{array}{c}\text { Transmission } \\
\text { Coefficient } \\
\text { (T) }\end{array}$ & $\begin{array}{c}K_{z} / Z \omega \\
\text { (specific stiffness) }\end{array}$ & $\begin{array}{c}K_{n z} / Z \omega \\
\text { (specific } \text { stiffness) }\end{array}$ & $\frac{K_{n z}}{K_{z}}$ & $\begin{array}{c}\text { Material } \\
\text { properties }\end{array}$ \\
\hline $0+$ & 0.9878 & 0.0243 & 0.078 & 0.038 & 0.5 & \multirow{2}{*}{$\begin{array}{l}\mathrm{Z}_{\mathrm{PZT}}=18 \\
\text { (MRayl) }\end{array}$} \\
\hline 5 & 0.9323 & 0.1308 & 0.194 & 0.084 & 0.43 & \\
\hline 15 & 0.9323 & 0.1307 & 0.194 & 0.084 & 0.43 & \multirow{2}{*}{$\begin{array}{l}Z_{\text {Steel }}=25 \\
\text { (MRayl) }\end{array}$} \\
\hline 25 & 0.9326 & 0.1303 & 0.193 & 0.085 & 0.44 & \\
\hline 35 & 0.9285 & 0.1378 & 0.20 & 0.086 & 0.43 & \multirow{2}{*}{$\begin{array}{c}\rho_{\mathrm{PZT}}=7.5 \\
\left(\mathrm{~g} / \mathrm{cm}^{3}\right)\end{array}$} \\
\hline 45 & 0.9165 & 0.1601 & 0.218 & 0.091 & 0.417 & \\
\hline 55 & 0.8970 & 0.1953 & 0.246 & 0.098 & 0.398 & \multirow{2}{*}{$\begin{array}{c}\rho_{\text {Steel }}=7.8 \\
\left(\mathrm{~g} / \mathrm{cm}^{3}\right)\end{array}$} \\
\hline 65 & 0.8892 & 0.2093 & 0.257 & 0.101 & 0.398 & \\
\hline 75 & 0.8874 & 0.2126 & 0.26 & 0.10 & 0.38 & $\omega=5 \mathrm{MHz}$ \\
\hline
\end{tabular}

Conversely, if the linear interfacial stiffness becomes very large, two contacting solids bond together very tightly and the hysteretic effect becomes relatively small.

\section{Conclusions}

An ultrasonic method for the measurement of hysteretic interfacial stiffness in solid-solid contact interface is proposed using pulse-echo signals from the interface. Mathematical derivation and formulation are made to determine the linear and hysteretic interfacial stiffness based on the reflection and transmission coefficients of the interface. Three consecutive echo signals are captured in experiment and analyzed to calculate the coefficients $\mathrm{R}$ and $\mathrm{T}$, by which the stiffness values are obtained without special phase measurement or advanced signal processing. For the demonstration of these characteristics of the hysteretic model, a simple contacting interface was made by joining two identical steel plates with bolt and examined by pulse-echo tests to measure the reflected waves from the contact interface. It was found from the experiment that the interfacial stiffness was successfully measured by the reflection and transmission coefficient and highly dependent on the pressure applied on the interface.

Experimental results showed that both the linear and hysteretic interfacial stiffness increased with the contact pressure (torque value 
applied to the specimen). However, the increasing rate of the linear stiffness was much higher than the hysteretic stiffness, so that it played a more role on the contact nonlinearity of the interface subject to high contacting pressure. It is concluded that the measurement technique of interfacial stiffness using the reflection and transmission coefficients may be useful to the characterization of nonlinear contact interfaces both in quantitative and qualitative sense because of its simplicity and ease of implementation.

\section{Acknowledgments}

This study was supported partly by Korea University of Technology and Education and Korea Institute of Energy Technology Evaluation and Planning (KETEP). The author expresses gratitude for the support.

\section{References}

[1] S. Biwa, A. Suzuki and N. Ohno, "Evaluation of interface wave velocity, reflection coefficients and interfacial stiffnesses of contacting surfaces," Ultrasonics, Vol. 43, pp. 495-502 (2005)
[2] S. Biwa, S. Hiraiwa and E. Matsumoto, "Experimental and theoretical study of harmonic generation at contacting interface," Ultrasonics, Vol. 44, pp. 1319-1322 (2006)

[3] S. Biwa, S. Hiraiwa and E. Matsumoto, "Stiffness evaluation of contacting surfaces by bulk and interface waves," Ultrasonics, Vol. 47, pp. 123-129 (2007)

[4] I. Y. Solodov, "Ultrasonics of non-linear contacts: propagation, reflection, and NDE applications," Ultrasonics, Vol. 36, pp. 383-390 (1998)

[5] J. Y. Kim, A. Baltazar and S. I. Rokhlin, "Ultrasonic assessment of rough surface contact between solids from elasto-plastic loading-unloading hysteresis cycle," Journal of the Mechanics and Physics of Solids, Vol. 52, pp. 1911-1934 (2004)

[6] J. Y. Kim, A. Baltazar, J. W. Hu and S. I. Rokhlin, "Hysteretic linear and nonlinear acoustic responses from pressed interfaces," International Journal of Solids and Structures, Vol. 43, pp. 6436-6452 (2006)

[7] N. Kim, K. -Y. Jhang, T. H. Lee, S. Y. Yang and Y. C. Chang, "Reflection and transmission of acoustic waves across nonlinear contact interface," Journal of the Korean Society for Nondestructive Testing, Vol. 28, No. 3, pp. 292-301 (2008) 